geht. Dass der Zerfall bei Actinosphärium und die Secretion bei Amblystoma auf der Anodenseite stattfindet, muss auch in die Wagschale geworfen werden.

Völlig unerwartet war für mich das in diesen Versuchen beobachtete Resultat, dass die Erregung stets an der Anodenseite des Centralnervensystems stattfindet. Eine derartige Abweichung vom Pflüger'schen Gesetz steht bis jetzt bei Wirbelthieren meines Wissens ganz vereinzelt da.

\title{
Nochmals über Kaiser's Theorie der Muskelzuckung.
}

Von

\section{Dr. Fr. Schenck.}

$\mathrm{K}$ a i s e ${ }^{1}$ ) hat die Hypothese aufgestellt, dass die contractilen Kräfte des Muskels seine bewegten Massentheilchen unter Umständen schleudern. Er gründet diese Hypothese auf folgende Beobachtung. Wenn man einen vom zuckenden Muskel bewegten Schreibhebel, der den Muskel möglichst wenig belastet, bei seiner Aufwärtsbewegung gegen einen festen Anschlag stossen lässt, so sinkt der Hebel sofort vom Anschlag zurück, falls der Anschlag mindestens in einer gewissen Höhe über der Ruhelage des Zeichenhebels eingestellt ist. Kaiser behauptet, das sofortige Absinken des Hebels vom Anschlag beruhe darauf, dass im Muskel keine contractile Kraft mehr wirke, sondern dass die geschleuderten Massentheilchen sich nur noch mit der ihnen von der Contractionskraft ertheilten lebendigen Kraft weiter bewegen und dass ihre Bewegungsrichtung sich beim Anprall des Hebels am Anschlag sofort umkehre, mithin der Hebel sofort absinke. Wenn der Anschlag so tief eingestellt sei, dass beim Anstossen des He-

1) Zeitschr. f. Biol. Bd. 33. S. 157. 
bels noch contractile Kraft im Muskel wirke, so bleibe der Hebel, durch die contractile Kraft gehalten, einige Zeit am Anschlag liegen. Als "zweiten Fusspunkt" bezeichnet Kaiser die Lage des unteren Muskelendes in dem Moment, wo die contractile Kraft des Muskels aufgehört hat auf die Bewegung der Massentheilchen zu wirken. Das sofortige Absinken des Hebels erfolgt demnach, wenn der Anschlag in oder ïber dem zweiten Fusspunkt eingestellt ist.

Ich habe nun gezeigt ${ }^{1}$ ), dass die Begründung, die $\mathrm{K}$ a i s e r seiner Hypothese giebt, einigen einfachen physikalischen Gesetzen widerspricht. Die physikalischen Gesetze habe ich erläntert durch schematische, mit einer entspannten Spiralfeder angestellte Versuche. Ich konnte den strengen Beweis bringen, dass die Beobachtungen, auf die Kaiser seine Hypothese gründet, nicht auf Schleuderung der Muskelmasse, sondern nur auf Schleuderung des Zeichenhebels beruhen.

Kaiser ${ }^{2}$ ) hat gegen meine Beweisfihrung Einwände gemacht. Es fällt mir die Aufgabe zu, seine Einwände zu entkräften.

I. Ehe ich auf die eigentlichen Einwände Kais er's eingehe, habe ich Folgendes zu bemerken. Kaiser beklagt sich, dass ich seine Mittheilung als "Theorie" bezeichne; er bestreitet mir die Berechtigung, die von ihm gefundenen ,Thatsachen" als Theorie zu bezeichnen.

Darauf ist zu erwidern: Selbst wenn es möglich wäre, dass die Schleuderung der Massentheilchen so zum Ausdruck kommt, wie Kaiser meint, wiirde aus dem, was er mitheilt, doch nur die Thatsache hervorgehen, dass Etwas geschleudert wird. Was dieses Etwas ist, dariuber geben seine Versuche keinen Aufschluss. Es könnte also recht wohl der Schreibhebel allein, und nicht die Muskelmasse geschleudert sein. Denn der positive Beweis ist nicht erbracht, dass Schleuderung des Hebels ausgeschlossen war. Also ist $K$ a i ser's Satz nur eine Vermuthung, keine Thatsache, und noch dazu eine recht unwabrscheinliche Vermuthung, weil manche Thatsachen es wahrscheinlich machen, dass die Bewegung der Massentheilchen im Muskel eine vollkommen gedämpfte ist. Ja ich habe diesem Satze noch zu viel Ehre angethan, wenn ich

1). Dies Archiv Bd. 63. S. 355.

2) Zeitschr. f. Biol. Bd. 33. S. 352. 
ihn Theorie nannte, denn eine Theorie sollte besser begründet sein, als dieser höchst unwahrscheinliche Satz.

II. Mein Haupteinwand gegen die Begründung, die $\mathrm{K}$ a is er seiner Hypothese giebt, ist folgender:

Wenn der Hebel am Anschlag anstösst, kann die Bewegungsrichtung der etwa geschleuderten Muskeltheilchen nur dann sich sofort umkehren, wenn sie in undehnbarer Verbindung mit dem Hebel sind. Ist die Verbindung dehnbar, so bewegen sich die Massentheilchen weiter und dehnen dabei das dehnbare Verbindungsstück; inzwischen muss der Hebel am Anschlag liegen bleiben, falls keine Eigenbewegungen des Hebels im Spiele sind. Die Muskeltheilchen sind mit dem Hebel in dehnbarer Verbindung durch den Muskel selbst. Der Befund $\mathrm{Ka}$ i s er's kann also nur durch Eigenbewegung des Hebels bedingt sein. Die Begründung, die Kaiser seiner Hypothesegiebt, ist physikalisch falseh.

$\mathrm{K}$ a i s e r nimmt offenbar diese meine Argumentation nicht ernst, denn er hat für sie keinen anderen Einwand übrig, als ein Ausrufungszeichen. Er sieht es also wohl als selbstverständlich an, dass meine Ueberlegungen unsinnig sind.

Ich habe mich bemüht, aus $\mathrm{K}$ a i s e r's Entgegnung heranszulesen, warum er meine Beweisfuhrung nicht ernst nimmt, und wie er sich den Vorgang der Umkehr der Bewegungsrichtung der Massentheilchen vorstellt. Die Aufklärung darüber entbält, wie ich sehe, eine Bemerkung, die auf S. 353 unten und 354 oben ${ }^{1}$ ) steht. Es wird da auseinandergesetzt, dass der Bewegung der Massentheilchen nach aufwärts zwei Kräfte entgegenwirken, nämlich erstens die mit dem Grade der Verkürzung zunehmenden, durch das Zusammendrücken geweckten elastischen Kräfte des Muskels und zweitens der durch den Anschlag verursachte ,abwärts wirkende Stoss"; „die von beiden Kräften bedingten Beschleunigungen addiren sich und der Hebel wird im Moment des Anschlages zurïckgestossen".

In dieser Vorstellung steckt ein Fehler. Der abwärts gerichtete Stoss erfolgt nicht, wenn Schleuderung des Zeichenhebels ausgeschlossen ist. Denn in diesem Falle bewirkt der Anschlag doch nur, dass die Aufwärtsbewegung des unteren Muskelendes

1) Zeitschr. f. Biol. Bd. 33 . 
plötzlich vollständig gehemmt wird, nicht aber, dass das untere Muskelende nach abwärts gezogen wird. Ebensowenig wie am unteren Muskelende wird auch an den Massentbeilchen des Muskels ein Zug nach unten durch den Anschlag ausgeübt.

Ein Massentheilchen des Muskels hat im Moment des Anschlags eine nach aufwärts gerichtete lebendige Kraft, die grösser ist, als die entgegenwirkende elastische Kraft des Mriskels. Desshalb strebt das Massentheilchen auch nach dem Anschlag, sich nach oben zu bewegen. Und es wird an dieser Aufwärtsbewegung nicht momentan gehindert, weil das Verbindungsstiick zwischen ihm und dem unteren Muskelende der debnbare Muskel selbst ist, der sich erst allmählich selbst anspannt und dabei einen langsam zunebmenden Zug auf das Massentheilchen ausübt, der in Verein mit der Druckelasticität das Massentbeilchen zur Umkelır der Bewegungsrichtung erst dann zwingt, wenn diese abwärts wirkenden Kräfte grösser als die lebendige Kraft des Massentheilchens geworden sind.

Ich betone daher, dass $\mathrm{K}$ a is e $\mathrm{r}$ keinen stichhaltigen Einwand gemacht hat gegen meinen Beweis, durch den ich die Fehler in seinen Ueberlegungen nachgewiesen habe.

Es dürfte im Interesse der Klarlegung unserer Controverse zweckmässig sein, nocbmals kurz die Frage zu formuliren, die wir verschieden beantworten. Sie lautet:

Erhält ein Massentheilchen des Muskels im Moment des Anschlags einen nach abwärts gerichteten Stoss, auch wenn Hebelschleuderung ausgeschlossen ist?

$\mathrm{K}$ a is er sagt ja, ich sage nein. Mit der Entscheidung dieser Frage steht oder fällt $\mathrm{Ka}$ aiser's Theorie.

III. Das bisher Gesagte würde genügen, um die Hypothese $\mathrm{K}$ a i ser's von neuem als falsch begründet zurückweisen zu können. Ich brauchte daher nicht nochmals auf meine Federversuche einzugehen, durch die ich die aus physikalischen Gesetzen gewonnene Vorstellung von der Bewegung der geschleuderten Massentheilchen anschaulich gemacht babe. Ich bin aber doch gezwungen, darauf einzugehen, weil $\mathrm{Ka}$ ise $\mathrm{r}$ an meinen schematischen Versuchen eine unberechtigte Kritik übt, durch die er die Beweiskraft dieser Versuche als zweifelhaft hinzustellen sucht, und die desshalb eine entschiedene Zurückweisung verdient.

In Anlehnung an eiven von Kaiser zur Erläuterung seiner 
Hypothese beschriebenen Versuch habe ich Versuche in folgender Art angestellt: Das untere Ende einer fest aufgehängten Spiralfeder wurde verknüpft mit einem leichten isotonischen Schreibhebel, der die Feder so gut wie gar nicht dehnt. Die Feder wurde nun durch Zug am unteren Ende gespannt, dann plötzlich entlastet. Die Feder entspannt sich und das untere Federende bewegt sich danach mit dem Schreibhebel iber die Lage heraus, die ihnen bei ruhender Feder zukommt, schwingt danach wieder zurück u. s. f. Bei der Aufwärtsbewegung stösst der Hebel gegen einen Anschlag, der über der Ruhelage des Hebels eingestellt ist. Nach Kaiser müsste nun der Hebel sofort vom Anschlag absinken, nach den Lehren der Physik dagegen einige Zeit am Anschlag liegen bleiben. Thatsächlich blieb der Hebel am Anschlag in den Versuchen einige Zeit liegen; meine Beweisführung erhielt also durch diesen einfachen Versuch eine experimentelle Bestätigung.

$\mathrm{K}$ aiser wendet gegen meine Versuche Folgendes ein: Der 2. Fusspunkt im Sinne seiner Theorie soll bei den Federversuchen angegeben werden durch die Lage des unteren Endes der ganz unbelasteten Feder ${ }^{1}$ ). Wenn in meinen Versuchen die Feder durch den Hebel so gut wie gar nicht gedehnt worden wäre, so bätte der Hebel bei ruhender Feder thatsächlich den 2. Fusspunkt angegeben. Nun behauptet $\mathrm{K}$ aiser, ich habe über die Belastung der Feder in meinen Versuchen falsche Angaben gemacht; die Feder müsse viel stärker belastet und gedehnt gewesen sein, so dass sogar der Anschlag unter dem „zweiten Fusspunkt" liege, aus folgenden beiden Gründen:

1. In der von mir mitgetheilten Figur liegt der Beginn der Entspannungseurve der Feder 11,5 mm unter der die Gleich-

1) Ich will diese Annahne hier zulassen, um die Discussion nicht ïbermässig auszudehnen; gegen diese Anמahme lassen sich aber Einwände machen. Der zweite Fusspunkt liegt in Wirklichkeit in den Federversuchen immer in der Horizontalen, die der Schreibhebel bei ruhender Feder zeichnet, einerlei ob die Feder stark oder schwach belastet ist. $\mathrm{K}$ a is e $\mathrm{r}$ nimmt an, dass der zweite Fusspunkt auch bei grösserer Belastung in die Lage des unteren Endes der unbelasteten Feder fällt, weil in der Feder so lange eine elastische verkürzende Kraft wirke bis dieser "Fusspunkt" überschritten ist. Das ist irrig, weil diese elastische Kraft compensirt und wirkungslos wird durch die grössere an der Feder hängende Last. 
gewichtslage angebenden Horizontalen, der höchste Punkt liegt $13,5 \mathrm{~mm}$ darüber. Das ist nach $\mathrm{K}$ a ise $\mathrm{r}$ einfach unmöglich, denn der Punkt, von dem die Feder bei der Entlastung ausgeht, muisste tiefer liegen, als der böchste erreichte Punkt.

Die Erklärung dieses Verhaltens liegt nun einfach darin, dass der Anfangstheil der Curve in der Figur nicht mit enthalten ist, und zwar weil die Feder vor der Entspannung so weit gedehnt war, dass die Schreibspitze sich von der Trommelfläche abgehoben hatte.

Dass die Curve nicht vollständig ist, musste $\mathrm{K}$ a i ser erkennen, aus folgenden Gründen:

a) Im Text meiner Abhandlung ist angegeben, dass die Figuren nur "die charakteristischen Theile" der Curven wiedergeben.

b) Wäre die Federcurve vollständig, so müsste sie links in der Figur mit einer horizontalen Linie beginnen, die in den aufsteigenden Schenkel überginge.

$\mathrm{Ka}$ iser durfte iuberdies die in Rede stehende Eigenthümlichkeit der Figur nicht zur Stütze seiner Ansicht heranziehen, dass meine Feder stark belastet gewesen ist; denn auch bei starker Belastung bätte der Beginn der Entspannungscurve weiter unter der Gleichgewichtslage liegen muissen, als der höchste Punkt der ersten S'chwingung iiber dieser Lage.

Uebrigens geht aus den weiteren Auseinandersetzungen Ka iser's auch hervor, dass er die Möglichkeit erkannt hat, dass die Figur die Curve nicht vollständig wiedergebe. Trotzdem scheut er sich nicht, meine Angaben auf Grund seiner oberflachlichen Kritik in's Lächerliche zu ziehen, indem er sagt, meine Feder wäre einem perpetnum mobile bedeutend überlegen. Ich weise diese Bemerkung als unstattbaft zuriick.

2. In meinen Federcurven ist der absteigende Schenkel anscheinend steiler, als der aufsteigende. Das kann - so sagt Kaiser - nur darauf beruhen, dass die Belastung meiner Feder eine nicht unbeträchtliche war. Hier hat $\mathrm{Ka}$ i s e r eine Grundregel der myographischen Methodik ausser Acht gelassen. Er bedenkt nicht, dass er über absolnte Werthe der Steilheit von Curven nur dann Aussagen machen kann, wenn er die Ordinatenrichtung kennt. Der Unterschied in der Steilheit beider Schenkel meiner Curven beruht nämlich nur auf der Entstellung der Curven, 
weil die Zeichenspitze in dem charakteristischen Theile bogenförmig und in der Richtung von rechts unten nach links oben sich bewegte.

Uebrigens habe ich schon vor Beginn unserer öffentlichen Discussion brieflich $\mathrm{K}$ a is e $\mathrm{r}$ die bestimmte Versicherung gegeben, dass in meinen Federversuchen der Ansehlag über dem 2. Fusspunkt liegt, als er gelegentlich unserer Correspondenz iiber meine Versuche mir schon den Einwand gemacht batte, dass bei mir der Anschlag unter dem 2. Fusspunkt liege. Trotzdem macht er mir jetzt den gleichen Einwand wieder öffentlich und stellt ganz entschieden die Richtigkeit meiner Angaben in Abrede!

Die für die Beurtheilung meiner Federversuche nöthigen Zahlenangaben will ich hier poch nachbolen. Die Spiralfeder war ans $0,7 \mathrm{~mm}$ dickem Stahldraht hergestellt, $100 \mathrm{~mm}$ lang, hatte 31 Windungen, der Durchmesser der einzelnen Windung betrug $12 \mathrm{~mm}$. Ihr Gewicht war $3 \mathrm{gr}$. Der Zeichenhebel war $130 \mathrm{~mm}$ lang, das untere Federende war $65 \mathrm{~mm}$ von der Achse entfernt am Hebel befestigt. Ueber der Achse, deren Radius $1,25 \mathrm{~mm}$ betrug, hing ein Faden, der ein Gewicht von $16 \mathrm{gr}$ trug. Die Belastung der Feder durch den Hebel allein betrug 0,15 gr, dazu kam noch die durch das angehängte Gewicht, das mit $0,3 \mathrm{gr}$ an der Feder wirkte. Im Ganzen betrug die Last der Feder also 0,45 gr. Belastete man die Feder direct mit $10 \mathrm{gr}$, so senkte sich die Zeichenspitze $3,5 \mathrm{~mm}$. Demnach wird die Feder durch eine Last von $0,45 \mathrm{gr}$ gedehnt um einen Betrag, der in der Vergrösserung durch die Hebelspitze rund $0,2 \mathrm{~mm}$ betrug. Der Hebel dehnt die Feder also nur um einen verschwindend kleinen Betrag, so dass man den 2. Fusspunkt in der von der ruhenden Feder gezeichneten Horizontalen anzunehmen berechtigt ist.

Ein Einwand könnte noch gegen die Richtigkeit dieser Annahme gemacht werden. Die aufgehängte Feder dehnt sich durch ihr eigenes Gewicht um einen bestimmten Betrag, wodurch eine wesentliche Verlagerung des 2. Fusspunkts bedingt sein könnte. Es fragt sich, wie gross der dadurch bewirkte Fehler sein kann. Die Dehnung, die die Feder durch ihr eigenes Gewicht erleidet, ist gleich der Hälfte des Produkts aus ibrem Gewicht und der durch die Gewichtseinheit bewirkten Dehnung, also in der Zeichnung: $\frac{3 \cdot 0,35}{2}=0,5 \mathrm{~mm}$. Der 2. Fusspunkt müsste also noch 
um 0,5 mm höher angenommen werden. Er liegt demnach im Ganzen $0,7 \mathrm{~mm}$ über der Horizontalen, die die Schreibspitze bei ruhender Feder gezeichnet hat. Der Anschlag, der sich etwa $6,5 \mathrm{~mm}$ iiber dieser Horizontalen befindet, liegt also bedeutend ii b e $\mathrm{r}$ dem 2. Fusspunkt.

Meine Versuche entsprechen also ganz den Anforderungen, die Kaiser stellt, und trotzdem sind sie in meinem Sinne ausgefallen.

Dass $\mathrm{K}$ a is er andere Resultate erhalten hat, als ich, liegt einfach daran, dass auch in seinen Federversuchen der Schreibhebel geschleudert worden ist. Auf die Möglichkeit einer Entstellung der Federcurven dureh Eigenbewegungen des Hebels habe ich schon in meiner Kritik der Hypothese Ka is er's aufmerksam gemacht. Ich babe mich auch durch Versuche davon iuberzengt, dass bei den Federversuchen leicht Hebelschleuderungen vorkommen.

IV. Ich war in der Kritik, die ich an Kaiser's Arbeit übte, zu dem Schlusse gekommen, dass die Curven Ka i ser's ,durch colossalen Wurf des Zeichenhebels vollständig entstellt" worden sind. Die Zumuthung, "mit einem so ungeheuerlichen Hebel" gearbeitet zu haben, weist Ka iser zurück. Dazu bemerke ich: Ich habe von einem ,ungeheuerlichen" Hebel nichts gesagt, sondern ich spreche nur ron ,colossalem Wurf". Es kann auch der leichteste Hebel stark geschleudert werden, wenn er nicht nach isotonischem Principe belastet, sondern unbelastet verwendet wird und wenn die Contractionsgeschwindigkeit sebr gross ist. Desshalb kommen gerade bei sehr geringer Belastung leicht enorme Schleaderungen vor. Das ist aligemein bekannt, scheint aber $\mathrm{K}$ a iser unbekannt zu sein.

Kaiser erklärt meine "Zumuthung" desshalb noch für ungerechtfertigt, weil ich gewusst habe, dass die Bestimmung des zweiten Fusspunkts auch mit Hebeln gelingt, die genau nach meinen Angaben gefertigt sind. Der Sachverhalt ist folgender: Gleich nach dem Erscheinen der ersten Abhandlung Kaiser's theilte ich ihm brieflich meine Bedenken mit and berief mich dabei auf meine Erfahrung, dass ich bei Verwendung eines möglichst nach isotonischem Principe arbeitenden sebr leichten Hebels nicht solche Erseheinungen gesehen habe, wie Kaiser. Er erwiderte, meine Befunde beruhten darauf, dass ich mit dem langsam zuckenden Esculentagastrocnemius gearbeitet habe; er liess sich 
einen Hebel nach meiner Angabe machen und erbielt damit bei Verwendung des schneller zuckenden Temporariagastrocnemius allerdings seinen „„weiten Fusspunkt". Als el mir dies mittheilte, habe ich ihm gleich eingewendet:

1. Dass ein Hebel, der vom langsam zuckenden Esculentamuskel nicht mehr geschleudert wird, recht wohl durch den schneller zuckenden Temporariamuskel gesehleudert werden kann.

2. Dass in der mir zugesandten neuen Curve der 2. Fusspunkt höher lag, als in den früher von $\mathrm{K}$ a i ser veröffentlichten, was durch geringere Schleuderung des Hebels bedingt sein könnte.

Ich weise desshalb den Vorwurf zurück, dass ich die Ergebnisse der Versuche mit dem nach meinen Angaben gefertigten Hebel ignorirt habe.

Ich hatte übrigens $\mathrm{K}$ a is er gerathen, einen möglichst leichten, jedoch nach isotonischem Principe belasteten Hebel za verwenden. Nun giebt er aber bei Figur 12 an, dass der Hebel u nbelas tet war. Sollte er den Versuch gar nicht nach meinen Angaben angestellt haben?

V. Auf einige nebensächliche Einwände, die mir Kaise r noch macht, gehe ich nicht ein, nicht weil ich darauf nichts erwidern könnte, sondern weil ihre Erörterung wenig Interesse hat.

Kaiser versucht schliesslich anch an seinen Muskelcurven zu zeigen, dass die von ihm auf Schleuderung im Muskel zurïckgefübrten Erscheinungen nicht auf Hebelschleuderung beruken können. Da nun aber die von ihm gegebene Erklärung physikalisch falsch ist, so $m$ üssen und $k$ önnen diese Erscheinungen anders erklärt werden, als er es thut. Aber darauf einzugehen hat keinen Zweck, weil diese Erscheinungen nunmehr ohne Interesse für die Wissenschaft sind. Auch die neueste Theorie, die $\mathrm{K}$ aiser auf seine Beobachtungen aufbaut, ist bedeutungslos.

VI. Der Nachweis, dass Ka i s e r's Versuchsresultate durch Hebelschleuderung bedingt sind, lässt sich iubrigens noch anders bringen. Wenn man den Scbreibhebel vor der Zuckung des Muskels bis zur Höhe des zweiten. Fusspunktes hebt und unterstiutzt bält, so dass er erst vom Maskel bewegt werden kann, wenn das untere Muskelende den zweiten Fusspunkt überschritten hat, so muisste nach $\mathrm{K}$ a i s e r die Curve, die der Hebel nun bei der Zuckung beschreibt, genau zusammenfallen mit dem über dem zweiten Fusspunkt liegenden Stück der Curve, die der Hebel ohne vor- 
herige Unterstïtzung gezeichnet hat; dagegen diirfte der im zweiten Fusspunkt unterstitzt gehaltene Hebel nicht mehr rom zuckenden Muskel höher gehoben werden, wenn $\mathrm{K}$ a i s e r's Versuchsresultate durch Hebelschleuderung bedingt sind.

Ich habe nun einige Versuche angestellt, in denen der Schreibhebel in oder iiber dem zweiten Fusspunkt unterstützt gehalten wurde. Es wurde dazu der indirect gereizte Gastrocnemius von Temporarien und Esculenten benutzt, an den nur ein leichter, den Muskel mit 0,2 gr belastender Hebel gehängt war. Als Verbindungsstiick zwischen Muskel und Hebel diente ein feines Haar. Der Hebel gab die Verkürzung in vierfacher Vergrösserung wieder. Um ein möglichst grosses Ueberschreiten des zweiten Fusspunkts zu erhalten, waren die Muskeln auf $24^{\circ}-290 \mathrm{C}$. erwärmt. Es wurde nun zuerst immer der zweite Fusspunkt durch Anschlagzuckungen bestimmt, dann eine Zuckung ausgeführt mit vorheriger Unterstützung des Hebels im zweiten Fusspunkt. D a ergab sich, dass der im zweiten Fusspunkt unterstüttgehaltene Hebel durcb die Zuckung nicht mehr gehoben wurde!

Damit die Versuche gelingen ist es übrigens nöthig, gewisse Vorsichtsmaassregeln zu beachten. Das Verbindungsstiuck zwischen Muskel und Hebel muss erstens wegen der Unterstützung biegsam sein und zweitens so leicht wie möglich, damit nicht Wurf des Verbindungsstiucks allein den unterstiutzten Hebel über den zweiten Fusspunkt bebt. Man darf zu diesen Versuchen daher nicht einen Draht als Verbindungsstück benutzen, wie es $\mathrm{K}$ a is e $\mathrm{r}$ in seinen Versuchen gethan hat. Mir erwies sich ein feines Haar als das zweckmässigste und leichteste Verbindungsstiick. Schliesslich muss die Möglichkeit bedacht werden, dass der Hebel über den zweiten Fusspunkt erhoben werden kann, wenn der frei hängende Muskel bei seiner Zuckung zu sehr seitlich hin und her wackelt. Es muss also darauf geachtet werden, dass der Muskel gerade herabhängt und bei der Zackung nicht zo sehr schwankt. 